\title{
UCRL-CONF-227155
}

LAW RENCE LIVERMORE N A TIO N A L LABORATORY

\section{Artificial Fluid Properties for Large-Eddy Simulation of Compressible Turbulent Mixing}

\author{
A. W. Cook
}

January 10, 2007

International Workshop on the Physics of Compressible Turbulent Mixing Paris, France July 17, 2006 through July 21, 2006 
This document was prepared as an account of work sponsored by an agency of the United States Government. Neither the United States Government nor the University of California nor any of their employees, makes any warranty, express or implied, or assumes any legal liability or responsibility for the accuracy, completeness, or usefulness of any information, apparatus, product, or process disclosed, or represents that its use would not infringe privately owned rights. Reference herein to any specific commercial product, process, or service by trade name, trademark, manufacturer, or otherwise, does not necessarily constitute or imply its endorsement, recommendation, or favoring by the United States Government or the University of California. The views and opinions of authors expressed herein do not necessarily state or reflect those of the United States Government or the University of California, and shall not be used for advertising or product endorsement purposes. 


\title{
Artificial Fluid Properties for Large-Eddy Simulation of Compressible Turbulent Mixing
}

\author{
Andrew W. Cook \\ Lawrence Livermore National Laboratory, Livermore CA 94550; awcook@llnl.gov
}

\begin{abstract}
An alternative methodology is described for Large-Eddy Simulation of flows involving shocks, turbulence and mixing. In lieu of filtering the governing equations, it is postulated that the large-scale behavior of an "LES" fluid, i.e., a fluid with artificial properties, will be similar to that of a real fluid, provided the artificial properties obey certain constraints. The artificial properties consist of modifications to the shear viscosity, bulk viscosity, thermal conductivity and species diffusivity of a fluid. The modified transport coefficients are designed to damp out high wavenumber modes, close to the resolution limit, without corrupting lower modes. Requisite behavior of the artificial properties is discussed and results are shown for a variety of test problems, each designed to exercise different aspects of the models. When combined with a 10th-order compact scheme, the overall method exhibits excellent resolution characteristics for turbulent mixing, while capturing shocks and material interfaces in crisp fashion.
\end{abstract}

Key words: shocks, turbulence, mixing, large-eddy simulation, hyperviscosity 


\section{Introduction}

The computational technique of Large-Eddy Simulation (LES) was invented by meteorologists in the 1960s [Smagorinsky, 1963, Lilly, 1967, Deardorff, 1970] as a means of representing very high Reynolds number flows on very coarse grids. The method stands in contrast to Direct Numerical Simulation (DNS), where the range of scales is limited to what can be fully represented on the mesh. The standard approach to deriving the LES equations is to apply a lowpass spatial filter to the Navier-Stokes equations in order to remove unresolvable scales of motion. This is typically done without specifying the exact form of the filter function. The LES filter however, must obey certain constraints; e.g., the transfer function must be identically zero for all wavenumbers above $k_{q}=\pi / \Delta$, where $\Delta$ is the grid spacing. This requirement stems from the fact that the computational grid does not support wavenumbers outside the Nyquist interval. If this requirement is not met, then the spectral content of the discrete variables will be aliased with respect to the filtered variables [Shannon, 1949]. Unfortunately, it is not possible to meet this Fourier-space cut-off with a filter that remains positive-definite in physical space; i.e., any filter satisfying the Nyquist limit will contain negative lobes in real space. This has the unpleasant side effect of generating filtered values outside physical bounds. For example, to ensure that mass fractions remain between zero and one, the real-space filter must strictly reside between zero and one. A more catastrophic potential of non-positive filters is that they can admit negative densities, which can cause a code to fail when, e.g., velocity is computed from momentum. This situation does not occur in the Reynolds-Averaged NavierStokes (RANS) approach, because ensemble averages are bounds preserving. 
Given that the aliasing and bounds constraints cannot both be met by a filter of convolution type, the question then is how to provide an inner-scale cutoff, such that the flow remains smooth with respect to the grid scale. One solution is to integrate the equations between grid points to directly generate a set of 2nd-order finite-difference equations. Such is the approach of Implicit Large-Eddy Simulation (ILES) [Schumann, 1975, Fureby and Grinstein, 2002, Margolin and Rider, 2002, 2005, Hickel et al., 2006]. This practice introduces two-dimensional cell-face averages as well as three-dimensional cell-volume averages, which must be related to each other. Typically, this is done implicitly via a numerical reconstruction scheme in one dimension, combined with operator splitting for multi-dimensional simulations. The truncation error of the numerical scheme can be designed to have desirable dissipative properties for turbulence. Much has been accomplished with ILES and the method appears to be growing in popularity; however, it is restricted to low-order upwind schemes with large dissipation and dispersion errors compared to spectral and compact schemes, which possess superior resolution characteristics for turbulence [Orszag and Patterson, 1972, Gottlieb and Orszag, 1977, Ghosal, 1995, Kravchenko and Moin, 1997]. Furthermore, since ILES dissipation is typically tied to differences in reconstruction on either side of a cell face, the method can be highly susceptible to grid imprinting.

The objective of this work is to introduce a methodology for LES which allows freedom to choose high-fidelity numerical schemes. Rather than modifying the governing equations or working directly with finite-difference equations, the fluid transport coefficients are altered to provide the sharpest possible cut-off near the Nyquist wavenumber. This work extends previous work on hyperviscosity [Cook and Cabot, 2004, 2005, Fiorina and Lele, in press] to include 
hyperconductivity for contact discontinuities and hyperdiffusivity for material interfaces. At the risk of crowding the LES field with yet another acronym, the method will be referred to as Artificial-Fluid Large-Eddy Simulation (AFLES). The outline of this paper is as follows. In Section 2 the governing equations for compressible turbulent mixing are laid out in strong conservation-law form. In Section 3, models are presented for the artificial fluid properties. In Section 4, the numerical solution technique is outlined. Section 5 describes results on a variety of test problems and Section 6 presents conclusions regarding the AFLES methodology. 


\section{Governing Equations}

Compressible flows comprised of $N$ miscible fluids can be described by the following equations:

$$
\begin{aligned}
& \frac{\partial \rho Y_{i}}{\partial t}+\nabla \cdot\left(\rho Y_{i} \mathbf{u}+\mathbf{J}_{i}\right)=0, \quad i=1,2, \ldots, N, \\
& \frac{\partial \rho \mathbf{u}}{\partial t}+\nabla \cdot[\rho \mathbf{u u}+p \underline{\boldsymbol{\delta}}-\underline{\boldsymbol{\tau}}]=0, \\
& \frac{\partial E}{\partial t}+\nabla \cdot[(E+p) \mathbf{u}-\underline{\boldsymbol{\tau}} \cdot \mathbf{u}+\mathbf{q}]=0,
\end{aligned}
$$

where $\rho$ is density, $Y_{i}$ is the mass fraction of species $i, \mathbf{u} \equiv \sum_{i=1}^{N} Y_{i} \mathbf{u}_{i}$ is velocity (with $\mathbf{u}_{i}$ being species velocity), $\mathbf{J}_{i} \equiv \rho Y_{i}\left(\mathbf{u}_{i}-\mathbf{u}\right.$ ) is a diffusive mass flux, $p$ is pressure, $\underline{\boldsymbol{\delta}}$ is the unit tensor, $\underline{\boldsymbol{\tau}}$ is the viscous stress tensor, $E \equiv \rho(e+\mathbf{u} \cdot \mathbf{u} / 2)$ is total energy (with $e$ being internal energy) and $\mathbf{q}$ is the heat conduction flux. For Newtonian fluids, the viscous stress tensor is

$$
\underline{\boldsymbol{\tau}}=\mu(2 \underline{\mathbf{S}})+\left(\beta-\frac{2}{3} \mu\right)(\nabla \cdot \mathbf{u}) \underline{\boldsymbol{\delta}},
$$

where $\mu$ is dynamic (shear) viscosity, $\beta$ is bulk viscosity and $\underline{\mathbf{S}}$ is the symmetric strain rate tensor,

$$
\underline{\mathbf{S}} \equiv \frac{1}{2}(\nabla \mathbf{u}+\mathbf{u} \nabla)
$$

where $\mathbf{u} \nabla$ denotes the transpose of $\nabla \mathbf{u}$. The conductive heat flux vector is described by Fourier's law,

$$
\mathbf{q}=-\kappa \nabla T
$$


where $\kappa$ is thermal conductivity and $T$ is temperature. The inter-diffusion energy flux and the diffusion-thermo (Dufour) energy flux are both neglected in (3). The diffusive mass fluxes can be approximated by

$$
\mathbf{J}_{i} \approx-\rho\left(D_{i} \nabla Y_{i}-Y_{i} \sum_{j=1}^{N} D_{j} \nabla Y_{j}\right),
$$

where $D_{i}$ is a species diffusion coefficient. In this Fickian approximation, pressure and temperature (Soret) forces are neglected and the multi-component fluid is regarded as a binary mixture of species $i$ and a complementary composite species composed of all other materials [Hirschfelder et al., 1954, Bird et al., 1960, Williams, 1985]. The last term in (7) is included to ensure that $\sum_{i=1}^{N} \mathbf{J}_{i}=0$.

For the test problems herein, all fluids are taken to be ideal gasses with the same constant ratio of specific heats. Pressure and temperature are thus computed as

$$
\begin{aligned}
& p=(\gamma-1) \rho e, \\
& T=(\gamma-1) e / R,
\end{aligned}
$$

where

$$
R=R_{o} \sum_{i=1}^{N} \frac{Y_{i}}{M_{i}}
$$

is the apparent gas constant with $R_{o}$ being the universal gas constant and $M_{i}$ being species molecular weight. 


\section{$3 \quad$ Artificial Fluid Properties}

The AFLES technique is based on adding grid-dependent components to the transport coefficients appearing in (4), (6) and (7); i.e.,

$$
\begin{aligned}
& \mu=\mu_{f}+\mu^{*}, \\
& \beta=\beta_{f}+\beta^{*}, \\
& \kappa=\kappa_{f}+\kappa^{*}, \\
& D_{i}=D_{f, i}+D_{i}^{*},
\end{aligned}
$$

where the $f$ subscripts denote fluid properties and the asterisks denote artificial properties. Like fluid properties, artificial properties are required to be both positive definite and frame invariant. Furthermore, they are required to carry over to the incompressible limit; e.g., viscosity cannot depend on sound speed. Unlike fluid properties, artificial properties are designed to vanish in smooth regions while providing strong damping near discontinuities. The following models satisfy all of these requirements:

$$
\begin{aligned}
& \mu^{*}=C_{\mu} \overline{\rho\left|\nabla^{r} S\right|} \Delta^{(r+2)}, \\
& \beta^{*}=C_{\beta} \overline{\rho\left|\nabla^{r} S\right|} \Delta^{(r+2)}, \\
& \kappa^{*}=C_{\kappa} \frac{\overline{\rho c_{s}}}{T}\left|\nabla^{r} e\right| \Delta^{(r+1)}, \\
& D_{i}^{*}=C_{D} \overline{\left|\nabla^{r} Y_{i}\right|} \frac{\Delta^{(r+2)}}{\Delta t}+C_{Y} \overline{\left\{\left[Y_{i}-1\right] H\left(Y_{i}-1\right)-Y_{i}\left[1-H\left(Y_{i}\right)\right]\right\}} \frac{\Delta^{2}}{\Delta t},
\end{aligned}
$$

where $S=(\underline{\mathbf{S}}: \underline{\mathbf{S}})^{1 / 2}$ is the magnitude of the strain rate tensor, $\Delta=$ $(\Delta x \Delta y \Delta z)^{1 / 3}$ is the local grid spacing, $c_{s}$ is sound speed, $\Delta t$ is the time step 
and $H$ is the Heaviside function. The polyharmonic operator, $\nabla^{r}$, denotes a series of Laplacians, e.g., $r=4$ corresponds to the biharmonic operator, $\nabla^{4}=\nabla^{2} \nabla^{2}$. This operator imbues the models with $k^{r}$ wavenumber damping, similar to a spectral vanishing viscosity [Tadmor, 1989, Karamanos and Karniadakis, 2000]. The overbar $(\bar{f})$ denotes a truncated-Gaussian filter, defined as

$$
\bar{f}(\mathbf{x})=\int_{-L}^{L} G(|\mathbf{x}-\boldsymbol{\xi}| ; L) f(\boldsymbol{\xi}) d^{3} \xi,
$$

where

$$
G(\zeta ; L)=\frac{e^{-6 \zeta^{2} / L^{2}}}{\int_{-L}^{L} e^{-6 \zeta^{2} / L^{2}} d \zeta}, \quad L=4 \Delta
$$

This filter eliminates cusps introduced by the absolute value operator, which in turn, ensures that the artificial transport properties are positive definite. For Cartesian grids, (19) can be applied sequentially along each grid line as

$$
\begin{aligned}
\bar{f}_{j}=\frac{3565}{10368} f_{j} & +\frac{3091}{12960}\left(f_{j-1}+f_{j+1}\right)+\frac{1997}{25920}\left(f_{j-2}+f_{j+2}\right) \\
& +\frac{149}{12960}\left(f_{j-3}+f_{j+3}\right)+\frac{107}{103680}\left(f_{j-4}+f_{j+4}\right) .
\end{aligned}
$$

The nondimensional constants appearing in (15)-(18) depend on $r$ (except for $C_{Y}$ ), but do not require adjustment from flow to flow. Setting $r=4$, the following values have been found to work well for a wide variety of test problems: $C_{\mu}=0.002, C_{\beta}=1, C_{\kappa}=0.01, C_{D}=0.003$ and $C_{Y}=100$. Except where otherwise noted, these values were used for all the the test problems herein.

The second term in the model for $D_{i}^{*}$ has been found very helpful in keeping mass fractions between 0 and 1 . It should be mentioned that for (18) to be 
Galilean invariant, $\Delta t$ must be chosen independently of $\mathbf{u}$. This is typically not the case when utilizing the CFL stability criterion. Nevertheless, (18) has been found to be quite useful in practice, since it removes ringing at material interfaces over a minimal number of timesteps. 


\section{Numerical Solution Technique}

\subsection{Spatial Differencing}

For the AFLES method described herein, all first derivatives comprising the gradient and divergence operators in the governing equations are computed according to the 10th-order compact scheme [Lele, 1992]

$$
\begin{aligned}
& \beta f_{j-2}^{\prime}+\alpha f_{j-1}^{\prime}+f_{j}^{\prime}+\alpha f_{j+1}^{\prime}+\beta f_{j+2}^{\prime} \\
& =a \frac{f_{j+1}-f_{j-1}}{2 \Delta}+b \frac{f_{j+2}-f_{j-2}}{4 \Delta}+c \frac{f_{j+3}-f_{j-3}}{6 \Delta},
\end{aligned}
$$

where $f_{j}^{\prime}$ represents the derivative of $f$ at node $j, \Delta$ is the spacing between nodes and

$$
\alpha=\frac{1}{2}, \beta=\frac{1}{20}, a=\frac{17}{12}, b=\frac{101}{150}, c=\frac{1}{100} .
$$

Similarly, all second derivatives $\left(f_{j}^{\prime \prime}\right)$, comprising the polyharmonic operators in the artificial fluid properties, are computed according to the 10th-order compact scheme

$$
\begin{aligned}
& \beta f_{j-2}^{\prime \prime}+\alpha f_{j-1}^{\prime \prime}+f_{j}^{\prime \prime}+\alpha f_{j+1}^{\prime \prime}+\beta f_{j+2}^{\prime \prime} \\
& =a \frac{f_{j+1}-2 f_{j}+f_{j-1}}{\Delta^{2}}+b \frac{f_{j+2}-2 f_{j}+f_{j-2}}{4 \Delta^{2}}+c \frac{f_{j+3}-2 f_{j}+f_{j-3}}{9 \Delta^{2}},
\end{aligned}
$$

where

$$
\alpha=\frac{334}{899}, \beta=\frac{43}{1798}, a=\frac{1065}{1798}, b=\frac{1038}{899}, c=\frac{79}{1798} .
$$




\subsection{Temporal Integration}

The governing equations are advanced in time by casting them all in the form $\dot{\Phi}=F$ and integrating via a five-step 4th-order Runge-Kutta (RK4) method derived by Kennedy et al. [1999]. The scheme is

$$
\begin{aligned}
& Q^{\eta}=\Delta t F^{\eta-1}+A^{\eta} Q^{\eta-1} \quad \eta=1, \ldots, 5 \\
& \Phi^{\eta}=\Phi^{\eta-1}+B^{\eta} Q^{\eta}
\end{aligned}
$$

where $\Delta t$ is the time step, $\eta$ is the RK4 subcycle, and $A^{\eta}$ and $B^{\eta}$ are:

$$
\begin{aligned}
& A^{1}=0 \\
& A^{2}=-6234157559845 / 12983515589748 \\
& A^{3}=-6194124222391 / 4410992767914 \\
& A^{4}=-31623096876824 / 15682348800105 \\
& A^{5}=-12251185447671 / 11596622555746 \\
& B^{1}=494393426753 / 4806282396855 \\
& B^{2}=4047970641027 / 5463924506627 \\
& B^{3}=9795748752853 / 13190207949281 \\
& B^{4}=4009051133189 / 8539092990294 \\
& B^{5}=1348533437543 / 7166442652324
\end{aligned}
$$


The fraction of $\Delta t$ for which the solution advances after each substep is:

$$
\begin{aligned}
& \eta=1 \Rightarrow 494393426753 / 4806282396855 \\
& \eta=2 \Rightarrow 4702696611523 / 9636871101405 \\
& \eta=3 \Rightarrow 3614488396635 / 5249666457482 \\
& \eta=4 \Rightarrow 9766892798963 / 10823461281321 \\
& \eta=5 \Rightarrow 1 .
\end{aligned}
$$

This particular RK4 scheme was chosen for its broad stability properties for both convective and viscous terms. Partial de-aliasing is accomplished by applying an 8th-order compact filter to the conserved variables $\rho Y_{i}, \rho \mathbf{u}$ and $E$ after each RK4 substep. The compact filter is designed to remove the top 1/10 of the wavenumbers in as sharp a manner as possible, such that results remain independent of the frequency of filter application (which depends on $\Delta t$ ). The filter stencil is

$$
\begin{array}{r}
\beta \hat{f}_{j-2}+\alpha \hat{f}_{j-1}+\hat{f}_{j}+\alpha \hat{f}_{j+1}+\beta \hat{f}_{j+2}=a f_{j}+\frac{b}{2}\left(f_{j-1}+f_{j+1}\right) \\
+\frac{c}{2}\left(f_{j-2}+f_{j+2}\right)+\frac{d}{2}\left(f_{j-3}+f_{j+3}\right)+\frac{e}{2}\left(f_{j-4}+f_{j+4}\right)
\end{array}
$$

where $\hat{f}_{j}$ is the filtered variable and

$$
\begin{gathered}
\alpha=0.66624, \quad \beta=0.16688, \quad a=0.99965, \quad \frac{b}{2}=0.66652 \\
\frac{c}{2}=0.16674, \quad \frac{d}{2}=4 \times 10^{-5}, \quad \frac{e}{2}=-5 \times 10^{-6} .
\end{gathered}
$$

This compact filter helps prevent the artificial fluid properties from becoming too large. For example, without this filter, $\beta^{*}$ can become extremely large in 
the vicinity of strong shocks, thus driving the viscously stable timestep to zero and bringing the simulation to a halt.

\subsection{Stability}

The maximum stable time step is limited not only by the inviscid CFL condition [Anderson et al., 1984],

$$
\Delta t_{C F L}=\operatorname{MIN}\left(\frac{|u|}{\Delta x}+\frac{|v|}{\Delta y}+\frac{|w|}{\Delta z}+c_{s} \sqrt{\frac{1}{\Delta x^{2}}+\frac{1}{\Delta y^{2}}+\frac{1}{\Delta z^{2}}}\right)^{-1}
$$

where $(u, v, w)=\mathbf{u}$, but also by the maximum viscosity, conductivity and diffusivity existing in the domain. Time scales associated with $\mu, \beta, \kappa$ and $D_{i}$ are:

$$
\begin{aligned}
& \Delta t_{\mu}=\operatorname{MIN}\left(\frac{\rho \Delta^{2}}{\mu}\right), \\
& \Delta t_{\beta}=\operatorname{MIN}\left(\frac{\rho \Delta^{2}}{\beta}\right), \\
& \Delta t_{\kappa}=\operatorname{MiN}\left(\frac{\rho c_{s}^{2} \Delta^{2}}{\kappa T}\right), \\
& \Delta t_{D}=\operatorname{MiN}\left(\frac{\Delta^{2}}{D_{i}}\right) .
\end{aligned}
$$

The simulation time step is chosen to be

$$
\Delta t=\operatorname{MIN}\left(\Delta t_{C F L}, 0.2 \Delta t_{\mu}, 0.2 \Delta t_{\beta}, 0.2 \Delta t_{\kappa}, 0.2 \Delta t_{D}\right)
$$




\section{Results}

The test problems in this section were selected to individually assess the efficacy of $\mu^{*}, \beta^{*}, \kappa^{*}$ and $D_{i}^{*}$ for capturing turbulent mixing with shocks and other discontinuities. All problems were run in exactly the same manner, using the default values of the model coefficients and setting $\mu_{f}=\beta_{f}=\kappa_{f}=D_{f, i}=0$. All problem specifications are in cgs units.

\subsection{Taylor-Green Vortex}

The ability of $\mu^{*}$ to accurately capture the evolution of the energy spectrum in high-Reynolds-number decaying turbulence has been previously demonstrated [Cook and Cabot, 2005]. As a further test of the ability of $\mu^{*}$ to resolve smallscale turbulence, we here consider the Taylor-Green vortex [Taylor and Green, 1937]. The initial conditions are:

$$
\begin{gathered}
\rho=1, \\
u=\sin (x) \cos (y) \cos (z), \\
v=-\cos (x) \sin (y) \cos (z), \\
w=0, \\
p=100+\{[\cos (2 z)+2][\cos (2 x)+\cos (2 y)]-2\} / 16, \\
\gamma=5 / 3 .
\end{gathered}
$$


where the pressure corresponds to the incompressible flow solution. The arbitrary constant of 100 is selected to make the Mach number very low, such that the incompressible solution can be used for comparison. As the flow evolves, the vortex stretches and bends, thus broadening the spectrum to include higher wavenumbers. Stretching and bending of vortex lines constitute key energy cascade mechanisms in turbulent flows. The computational domain is a triply-periodic $(2 \pi)^{3}$ box on a $64^{3}$ grid. Figure 1 shows normalized total enstrophy, i.e., $\Omega(t) / \Omega(0)$, where

$$
\Omega(t)=\frac{1}{2} \int_{V} \boldsymbol{\omega} \cdot \boldsymbol{\omega} d V, \omega=\nabla \times \mathbf{u} .
$$

Two ILES schemes are included for comparison: a Piecewise Liner MUSCLE Direct Eulerian (PLMDE) scheme [Colella, 1985] and an Arbitrary Lagrangian Eulerian (ALE) method [Marinak et al., 2001]. The theoretical result [Brachet et al., 1983], accurate up to about $t=3.5$, is plotted in order to assess the ability of each method resolve small-scale vortex dynamics. Analysis based on Padé approximants and the behavior of the analyticity strip, predicts $\Omega(t)$ will become far too large to capture on a such a coarse grid [Morf et al., 1980]; nevertheless, the ability of a scheme to track the enstrophy curve, as well as the maximum enstrophy that a scheme is able to generate, provide stringent tests of resolving power. The AFLES method is seen to have much better resolution characteristics that either of the ILES methods. For further comparison, the normalized kinetic energy, i.e., $K(t) / K(0)$, where

$$
K(t)=\frac{1}{2} \int_{V} \rho \mathbf{u} \cdot \mathbf{u} d V
$$

is plotted in Fig. 2. The AFLES method is better able to preserve kinetic energy as it cascades toward small scales. 


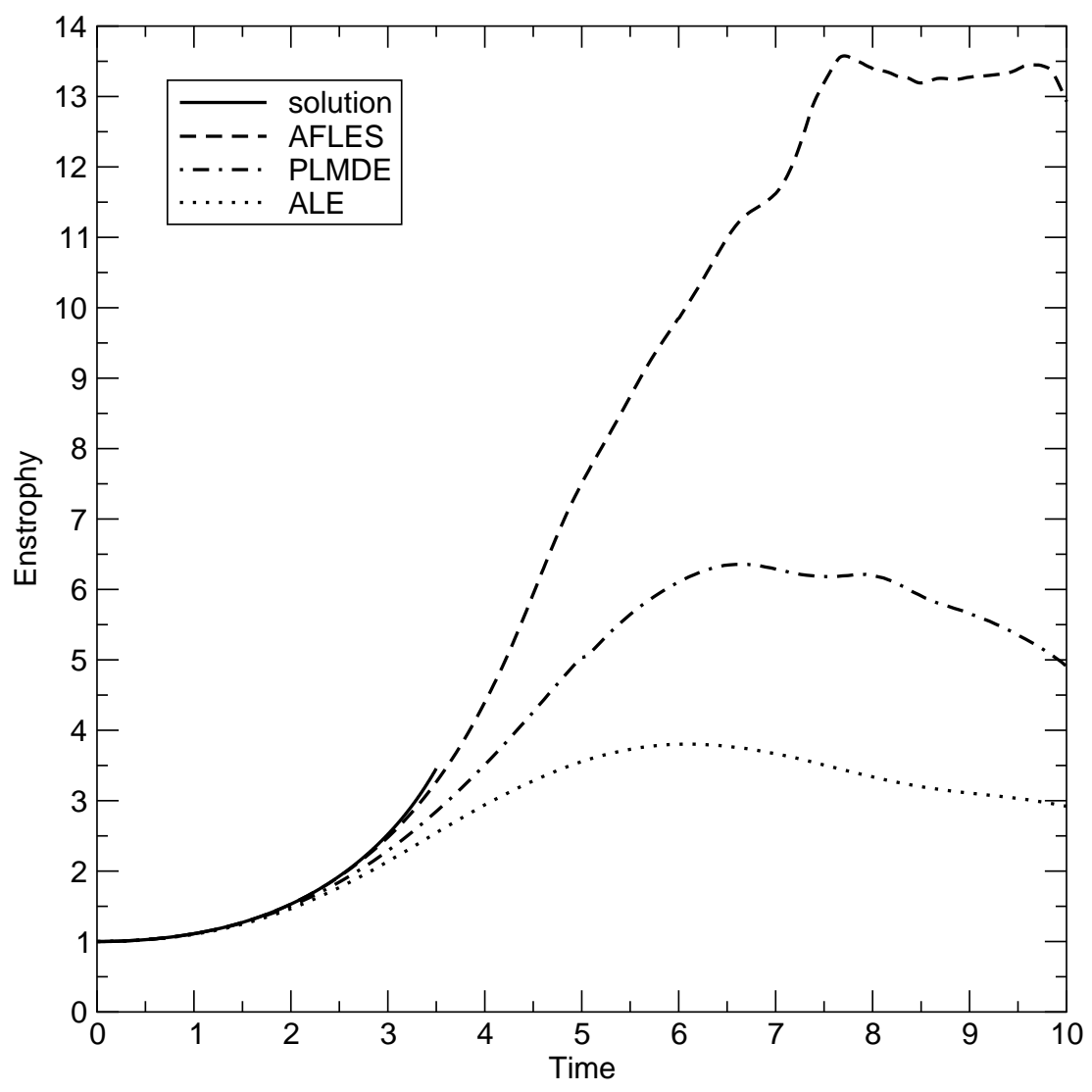

Fig. 1. Normalized enstrophy versus time for the Taylor-Green vortex. Simulations were conducted in a $2 \pi^{3}$ periodic box using $64^{3}$ grid points.

\subsection{Sedov Blast Wave}

In previous studies, the model for $\beta^{*}$ was demonstrated to provide $\mathcal{O}(r)$ convergence for smooth flow [Cook and Cabot, 2004] and capture shocks in approximately 3 grid points, regardless of shock strength [Fiorina and Lele, in press]. In order to assess the ability of $\beta^{*}$ to capture shocks in a sharp and monotonic fashion in multiple dimensions, we consider the Sedov-Taylor-von Neumann blast wave [Sedov, Taylor, 1950, Landau and Lifshitz, 1959]. The 


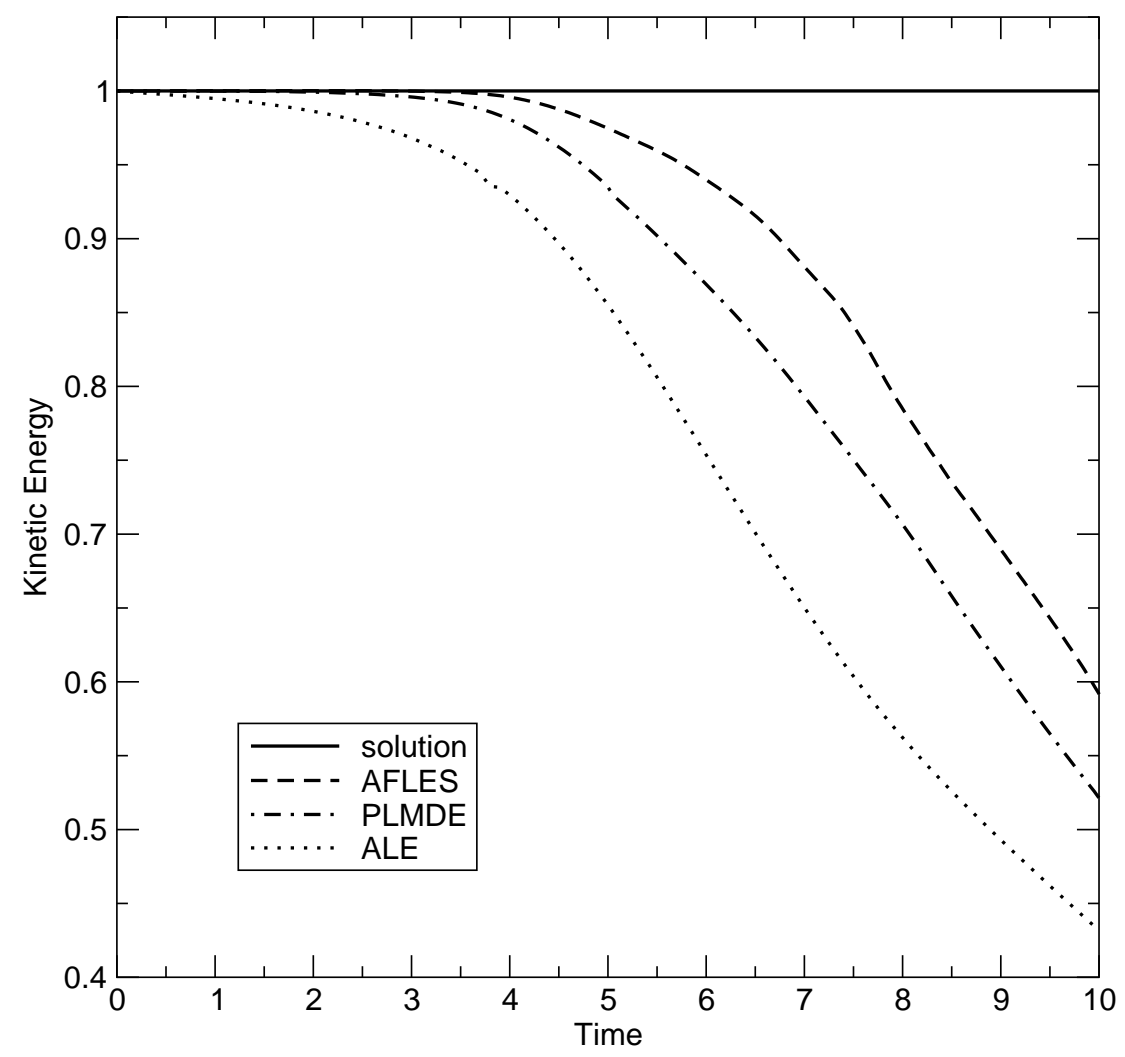

Fig. 2. Normalized kinetic energy versus time for the Taylor-Green vortex. Simulations were conducted in a $2 \pi^{3}$ periodic box using $64^{3}$ grid points.

initial conditions are:

$$
\begin{gathered}
\rho=1, \\
\mathbf{u}=0, \\
e=0.1528415451 \exp ^{\left(-\mathcal{R}^{2} / \epsilon^{2}\right)} / \epsilon^{3}, \quad \epsilon=0.04, \\
\gamma=1.4,
\end{gathered}
$$

where $\mathcal{R}=\sqrt{x^{2}+y^{2}+z^{2}}$ is radius. The exponential is employed to approximate a delta function on a discrete mesh. The computational domain consists 
of a uniform Cartesian mesh centered about the origin. Simulations were carried out at two resolutions, using a $128^{3}$ grid $(\Delta=0.02)$ and a $256^{3}$ grid $(\Delta=0.01)$. The results for the density field are displayed in Fig. 3, where the scheme is seen converging to the correct solution. A challenging aspect of this problem is that the blast wave leaves behind a vacuum at the origin. Nevertheless, the AFLES method captures the shock in a crisp angle-independent fashion. Furthermore, integration over the box shows mass, momentum and total energy to be conserved to more than 8 digits.

\subsection{Contact Discontinuities}

The need to treat temperature discontinuities independently of shocks was pointed out by Fiorina and Lele [in press]. Initial conditions, radiation effects and chemical reactions are all capable of producing steep temperature gradients in a smoothly varying velocity field. In order to assess the ability of $\kappa^{*}$ to capture such gradients, we consider a quiescent fluid containing a jump in entropy. The initial conditions are:

$$
\begin{gathered}
\rho=0.1+0.9 H(x), \\
\mathbf{u}=0, \\
p=1 \times 10^{10}, \\
\gamma=5 / 3 .
\end{gathered}
$$

For a nonconducting fluid, this one-dimensional flow should remain fixed at its initial state. Figure 4 shows results of the AFLES method with and without the thermal conductivity model. Without the model $\left(C_{\kappa}=0\right)$, Gibbs oscillations, 
introduced by the compact difference and filter stencils, remain undamped. By including the model, the oscillations are removed and the discontinuity is spread over 4 to 5 grid points. Once the discontinuity has been sufficiently smoothed, the model essentially turns off, preventing further spreading.

Given that $\kappa^{*}$ is necessary for treating jumps in temperature (which remain unaffected by $\mu^{*}$ and $\beta^{*}$ ), the question arises as to how to determine an upper bound on $C_{\kappa}$ to ensure that the model is not excessively dissipative. To answer this question, we turn to the one-dimensional Shu-Osher problem [Shu and Osher, 1989]. The initial conditions are:

$$
\begin{gathered}
\rho=3.857143, p=10.33333, u=2.629369 \text { for } x<-4 \\
\rho=1+0.2 \sin (5 x), p=1, u=0 \text { for } x \geq-4 \\
\gamma=1.4
\end{gathered}
$$

As the shock propagates into the sinusoidal density field, it leaves a steeply undulating flow in the post-shock region. The post-shock undulations are extremely sensitive to thermal conduction, as demonstrated in Fig. 5, which shows AFLES results for three different values of $C_{\kappa}$. With the default value of $C_{\kappa}=0.01$, the post-shock solution remain nearly identical to the case with no artificial thermal conductivity $\left(C_{\kappa}=0\right)$. Increasing $C_{\kappa}$ to 0.1 however, causes noticeable damping of the undulations.

\subsection{Material Interfaces}

For flows involving more than one fluid, artificial diffusivity is necessary to avoid ringing in the mass fractions. In order to assess the ability of $D_{i}^{*}$ to 
capture material interfaces, we first consider the simple case of two adjacent fluids in thermodynamic equilibrium. The initial conditions are:

$$
\begin{gathered}
\rho=1, \\
\mathbf{u}=0, \\
p=1 \times 10^{10}, \\
Y_{1}=H(x), \\
Y_{2}=1-Y_{1}, \\
\gamma=5 / 3 .
\end{gathered}
$$

For immiscible fluids, this one-dimensional problem should remain fixed at its initial state. Numerically, the compact stencils generate Gibbs oscillations about the step function, which are strongly damped within the first few timesteps. This process is illustrated in Fig. 6, which displays $Y_{1}$ versus $x$ at $t=0, \Delta t, 2 \Delta t, 3 \Delta t, 10 \Delta t$ and $1000 \Delta t$. The diffusivity model eliminates virtually all ringing within the first 3 timesteps and then effectively turns off, leaving the interface nearly frozen, as evidenced by the profile after a thousand steps. This behavior is independent of the grid spacing and the size of the timestep. The artificial diffusivity spreads interfaces over about 4 grid points within 3 or 4 timesteps and then vanishes to prevent further spreading.

In order to test the diffusivity model in the presence of strain, we consider the deformation of a circular blob of material in a time-reversing vortex. Following Leveque [1996] and Rider and Kothe [1998] the velocity field is prescribed for 
all time as

$$
\begin{gathered}
u=-\sin ^{2}(\pi x) \sin (2 \pi y) \cos \left(\pi t / t_{p}\right), \\
v=\sin ^{2}(\pi y) \sin (2 \pi x) \cos \left(\pi t / t_{p}\right), \\
t_{p}=4,
\end{gathered}
$$

with the blob of material initialized as

$$
\begin{gathered}
Y_{1}=\{1+\operatorname{erf}[(\mathcal{R}-0.15) / \epsilon]\} / 2, \\
\mathcal{R}=\left[(x-0.5)^{2}+(y-0.75)^{2}\right]^{1 / 2}, \\
\epsilon=0.01 .
\end{gathered}
$$

The $\epsilon$ parameter imparts a thickness to the material interface to avoid initial ringing. This temporally periodic flow stretches the circular blob into a spiral, then returns to its initial conditions whenever $t$ reaches integer multiples of $t_{p}$. This process is illustrated in Fig. 7 , which shows the blob repeatedly spiraling inward, then returning to its initial state. The straining action causes the interface to thin down to about 3 or 4 grid points, at which point the artificial diffusivity turns on to prevent further thinning (which would generate ringing). The amount of mixed fluid produced through artificial diffusion depends on $\epsilon, t_{p}$ and $\Delta$; however, for any given set of these parameters, the model becomes active only where necessary to prevent spurious oscillations in the mass fractions. 


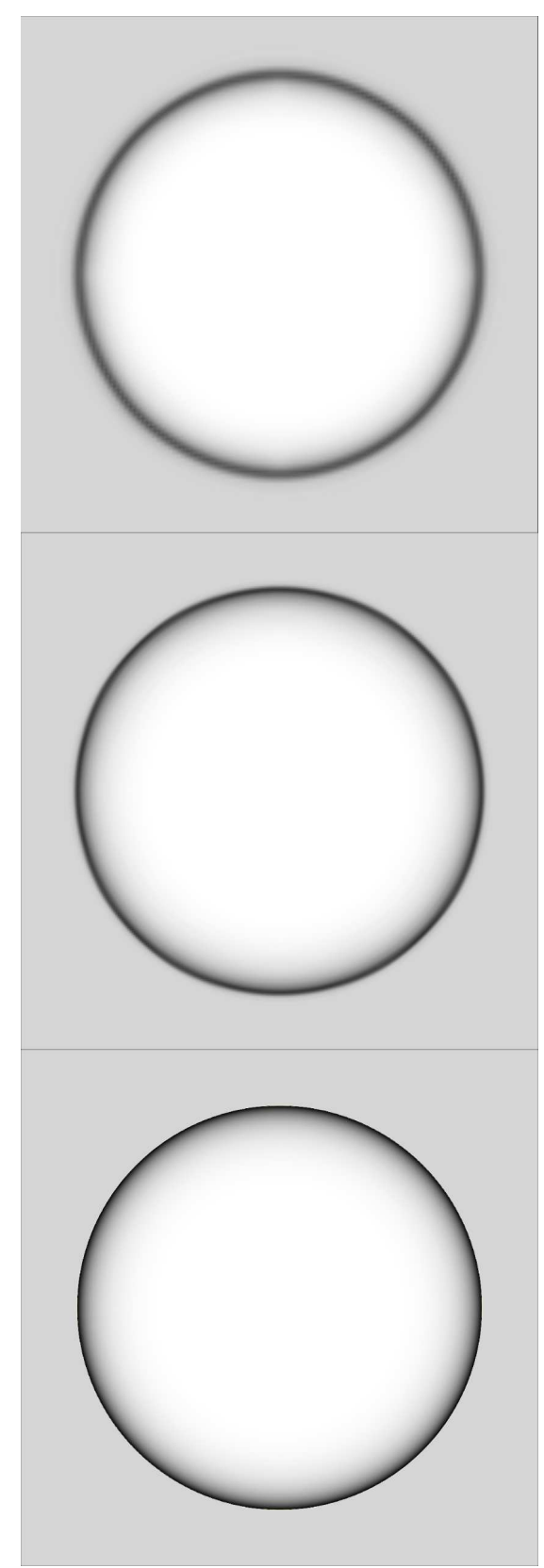

Fig. 3. Density on the $z=0$ plane for the Sedov-Taylor-von Neumann blast wave at $t=1$. White corresponds to $\rho=0$, whereas black corresponds to $\rho=6$. The top image is AFLES with $\Delta=0.02$, the middle image is AFLES with $\Delta=0.01$ and the bottom image is the solution. 


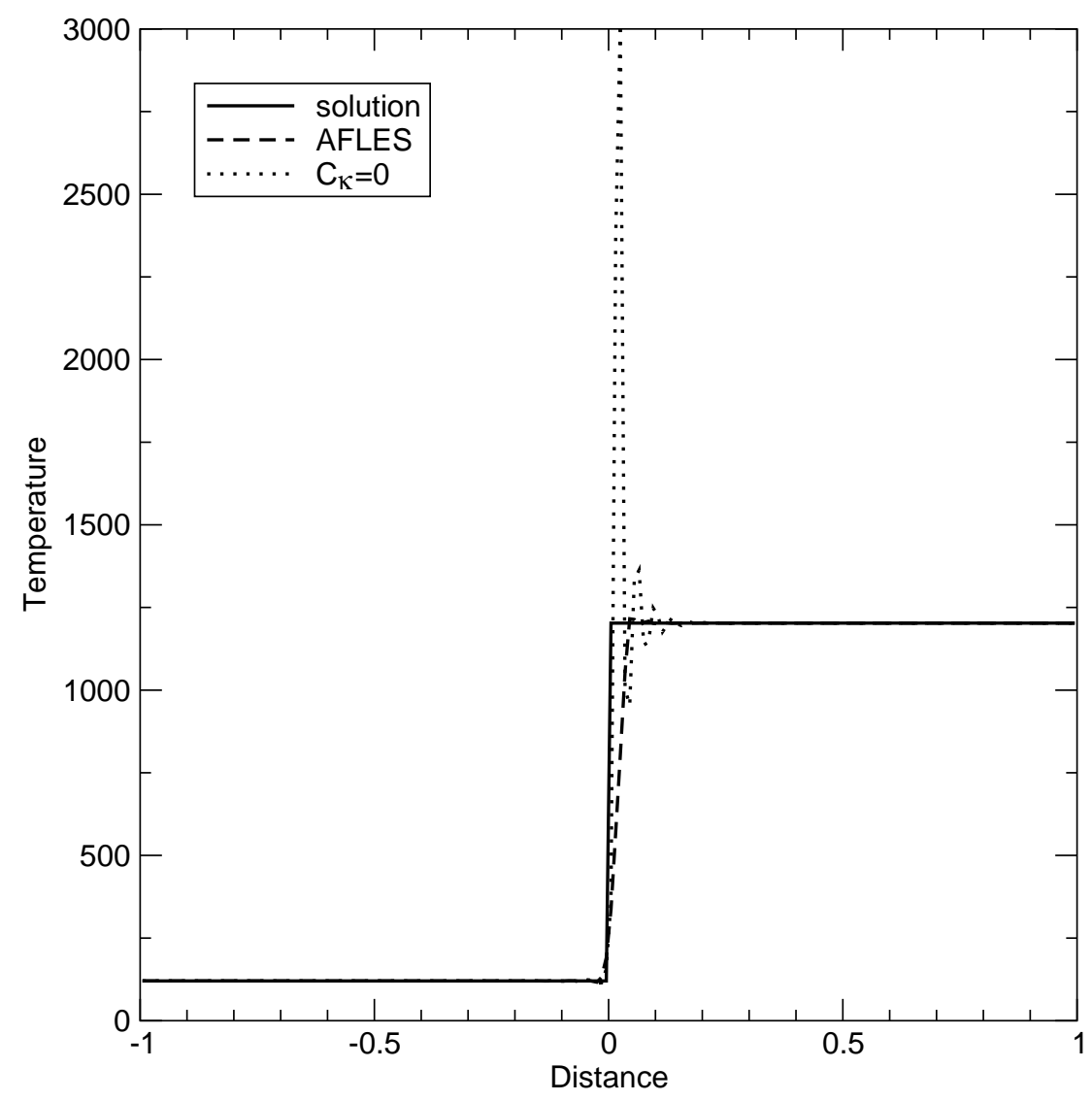

Fig. 4. Temperature at $t=1 \times 10^{-5}$ for the quiescent contact discontinuity using a grid resolution of $\Delta=0.01$. 


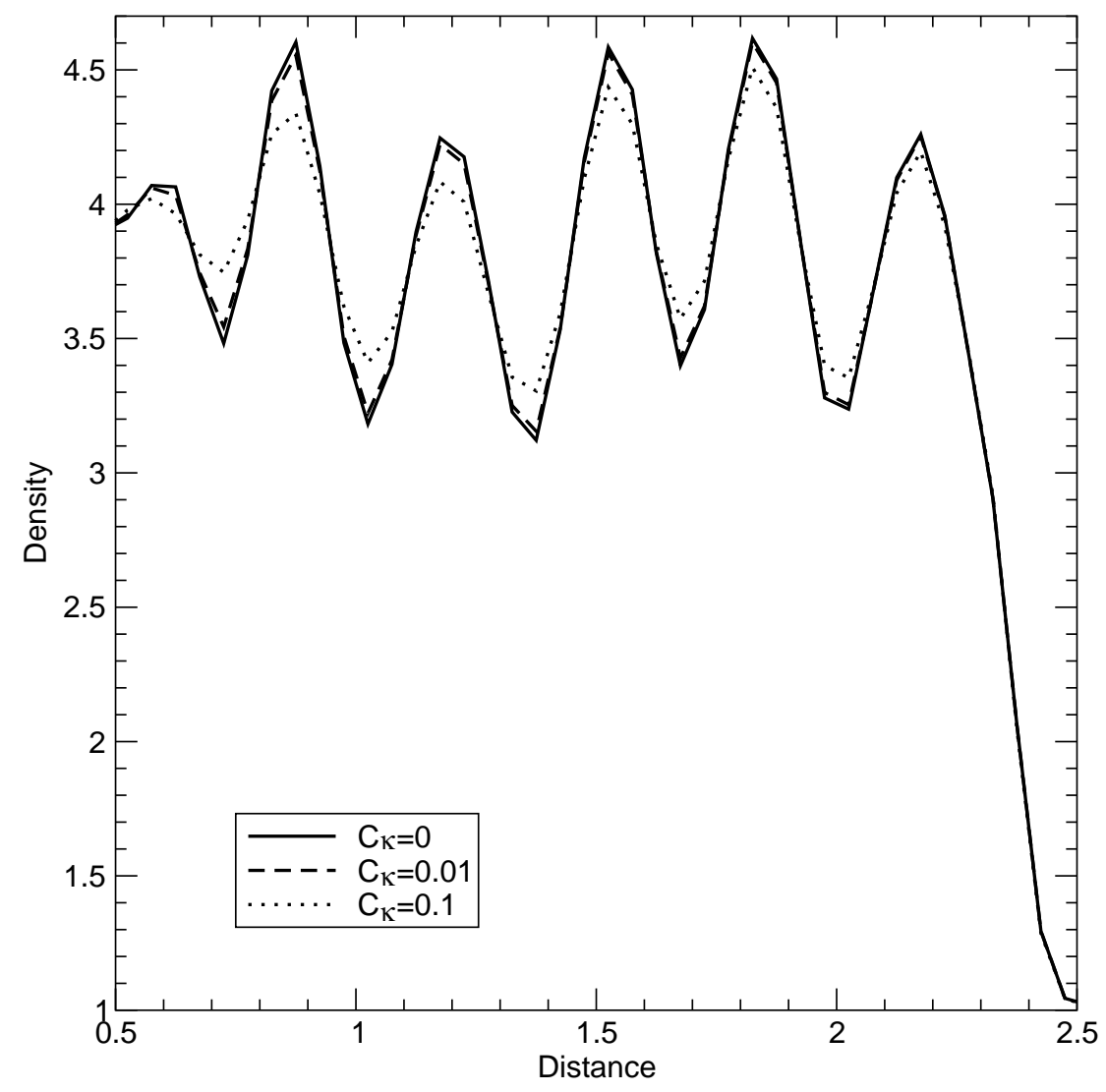

Fig. 5. Density field for the Shu-Osher problem at $t=1.8$ using a grid spacing of $\Delta=0.05$. 


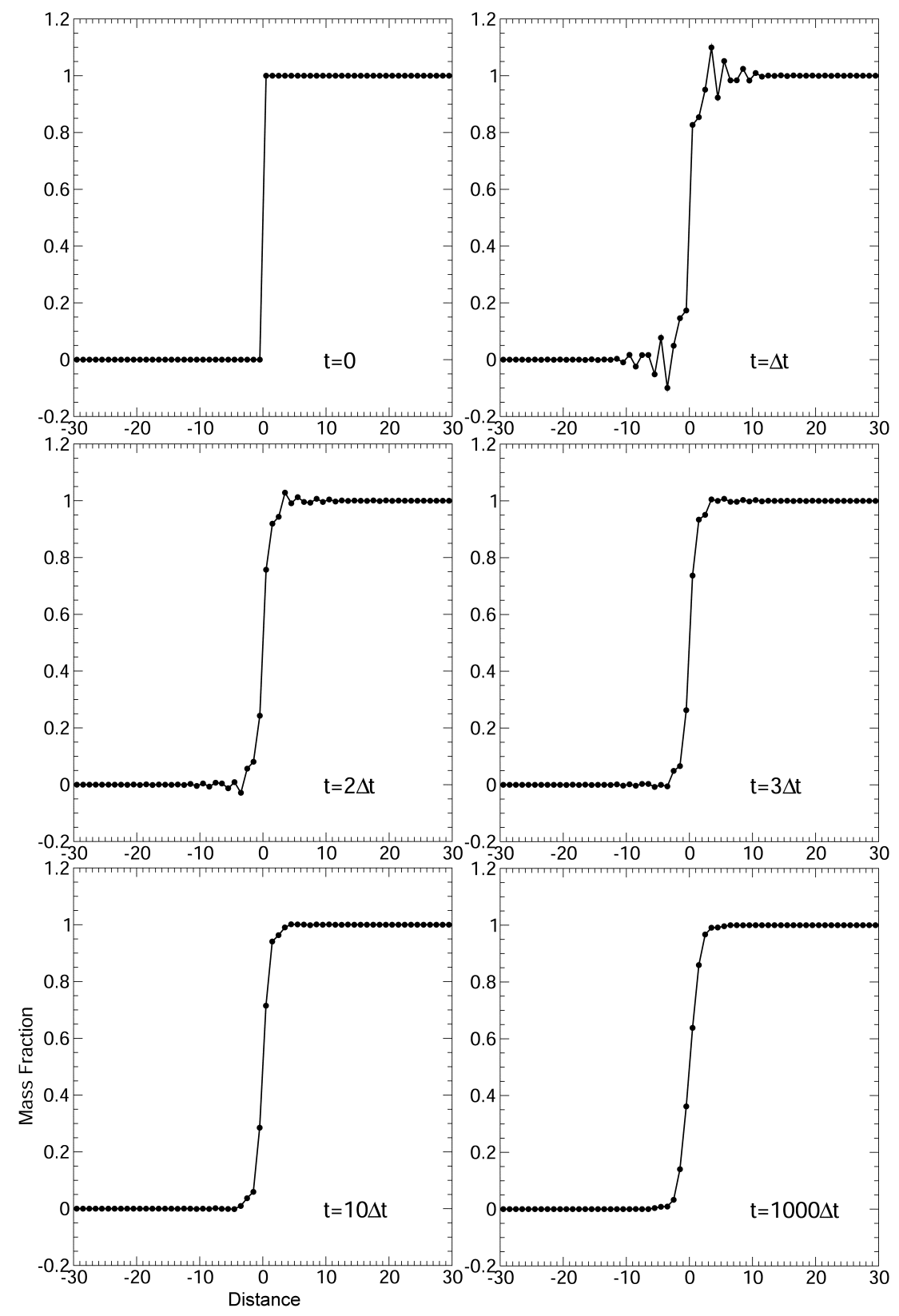

Fig. 6. Evolution of mass fraction interface for two adjacent quiescent fluids. 


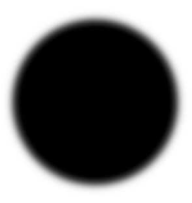

$\mathrm{t}=0$

$\mathrm{t}=2$

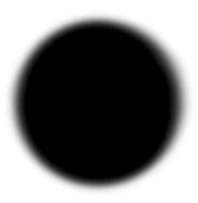

$t=4$
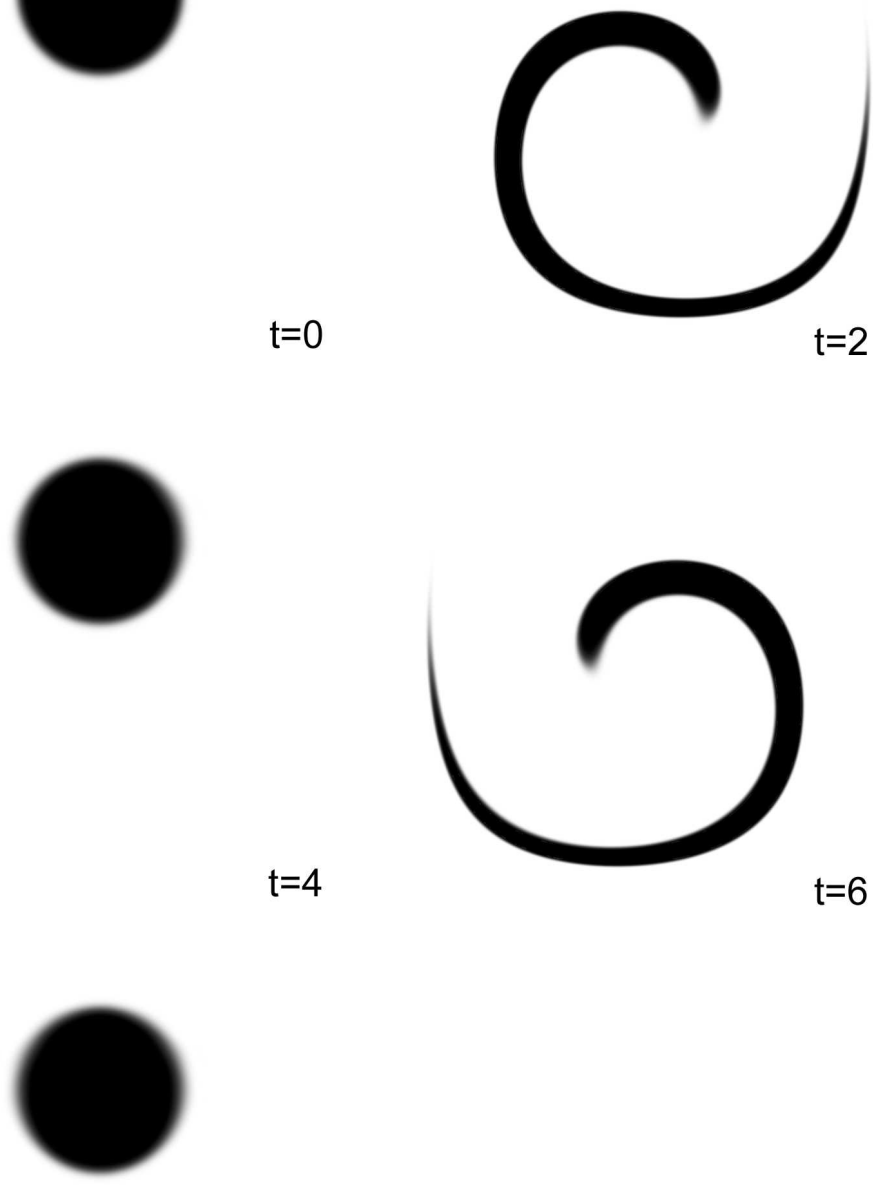

$t=8$

Fig. 7. Scalar advection in a time-reversing vortex. 


\section{Conclusions}

Spectral-like models have been introduced for the shear viscosity, bulk viscosity, thermal conductivity and species diffusivity of an artificial, "LES", fluid. The models impart a high wavenumber, $\kappa^{r}$, weighting to the numerical dissipation, thus preserving fidelity at resolved wavenumbers. The models act like switches, turning on only where fields are insufficiently smooth with respect to the grid scale. When the models are combined with high-wavenumberpreserving schemes, a dealiasing filter is required to meet stability constraints and help localize the numerical dissipation. The stencils for the filters and derivative operators in the AFLES scheme are all purely centered; hence, the method is minimally dissipative.

The AFLES scheme outperforms standard ILES methods in resolving the enstrophy field of the Taylor-Green vortex. By including the bulk viscosity term in the governing equations, the scheme is able to capture shocks without excessive damping of vorticity. Artificial thermal conductivity helps remove ringing at heat fronts, while artificial diffusivity helps keep mass fractions within bounds. The five empirical coefficients appearing in the models have been found to exhibit fairly universal behavior; i.e., they appear to work for a wide variety of flows without adjustment. The AFLES method presented herein provides a simple and effective approach to LES when the Reynolds number, Mach number and/or Schmidt number of a flow is beyond the reach of DNS. 


\section{Acknowledgements}

The author is grateful to Dr. W. H. Cabot for assistance in writing the code and Dr. P. L. Miller for feedback on the manuscript. This work was performed under the auspices of the U.S. Department of Energy by the University of California Lawrence Livermore National Laboratory under contract No. W7405-Eng-48.

\section{References}

D. A. Anderson, J. C. Tannehill, and R. H. Pletcher. Computational Fluid Mechanics and Heat Transfer. Hemisphere Publishing Corporation, 1984.

R. B. Bird, W. E. Stewart, and E. N. Lightfoot. Transport Phenomena. John Wiley and Sons, 1960.

M. E. Brachet, D. I. Meiron, S. A. Orszag, B. G. Nickel, R. H. Morf, and U. Frisch. Small-scale structure of the Taylor-Green vortex. J. Fluid Mech., 130:411-452, 1983.

P. Colella. A direct Eulerian MUSCL scheme for gas dynamics. SIAM J. Sci. Stat. Comput., 6:104-117, 1985.

A. W. Cook and W. H. Cabot. A high-wavenumber viscosity for highresolution numerical methods. J. Comput. Phys., 195:594-601, 2004.

A. W. Cook and W. H. Cabot. Hyperviscosity for schock-turbulence interactions. J. Comput. Phys., 203:379-385, 2005.

J. W. Deardorff. A numerical study of three-dimensional turbulent channel flow at large Reynolds numbers. J. Fluid Mech., 41:453-480, 1970.

B. Fiorina and S. K. Lele. An artificial nonlinear diffusivity method for supersonic reacting flows with shocks. J. Comput. Phys., in press. 
C. Fureby and F. F. Grinstein. Large eddy simulation of high-Reynoldsnumber free and wall-bounded flows. J. Comput. Phys., 181:68-97, 2002.

S. Ghosal. An analysis of numerical errors in large eddy simulations of turbulence. Annual Research Briefs, Center for Turbulence Research, Stanford University, 1995.

D. Gottlieb and S. A. Orszag. Numerical Analysis of Spectral Methods. Capital City Press, Montpelier, 1977.

S. Hickel, N. A. Adams, and J. A. Domaradzki. An adaptive local deconvolution method for implicit LES. JCP, 213:413-436, 2006.

J. O. Hirschfelder, C. F. Curtiss, and R. B. Bird. Molecular Theory of Gases and Liquids. Wiley, New York, 1954.

G-S. Karamanos and G. E. Karniadakis. A spectral vanishing viscosity method for large-eddy simulations. J. Comput. Phys., 163:22-50, 2000.

C. A. Kennedy, M. H. Carpenter, and R. M. Lewis. Low-storage, explicit Runge-Kutta schemes for the compressible navier-stokes equations. ICASE Report No. 99-22, 1999.

A. G. Kravchenko and P. Moin. On the effect of numerical errors in large eddy simulations of turbulent flows. J. Comput. Phys., 131:310-322, 1997.

L. D. Landau and E. M. Lifshitz. Fluid Mechanics. Addison-Wesley, Reading, Mass., 1959.

S. K. Lele. Compact finite difference schemes with spectral-like resolution. J. Comput. Phys., 103:16-42, 1992.

R. J. Leveque. High-resolution conservative algorithms for advection in incompressible flow. SIAM J. Numer. Anal., 33:627, 1996.

D. K. Lilly. The representation of small-scale turbulence in numerical simulation experiments. In Proc. IBM Sci. Comput. Symp. Environ. Sci., pages 195-210. IBM Data Process. Div., 1967. 
L. G. Margolin and W. J. Rider. A rationale for implicit turbulence modeling. Int. J. Numer. Meth. Fluids, 39:821-841, 2002.

L. G. Margolin and W. J. Rider. The design and construction of implicit LES models. Int. J. Numer. Meth. Fluids, 47:1173-1179, 2005.

M. M. Marinak, G. D. Kerbel, N. A. Gentile, O. Jones, D. Munro, S. Pollaine, T. R. Dittrich, and S. W. Haan. Three-dimensional HYDRA simulations of National Ignition Facility targets. Phys. Plasmas, 8:2275-2280, 2001.

R. H. Morf, S. A. Orszag, and U. Frisch. Spontaneous singularity in threedimensional, inviscid, incompressible flow. Phys. Rev. Lett., 44:572-575, 1980.

S. A. Orszag and G. S. Patterson. Numerical simulation of three-dimensional homogeneous isotropic turbulence. Phys. Rev. Lett., 28:76-79, 1972.

W. J. Rider and D. B. Kothe. Reconstructing volume tracking. J. Comput. Phys., 141:112-152, 1998.

U. Schumann. Subgrid-scale model for finite-difference simulations of turbulence in plane channels and annuli. J. Comput. Phys., 18:376-404, 1975.

L. I. Sedov. Similarity and Dimensional methods in Mechanics, Fourth Ed. Academic Press, New York.

C. E. Shannon. Communication in the presence of noise. Proceedings of the IRE, 37:10-21, 1949.

C.-W. Shu and S. J. Osher. Efficient implementation of essentially nonoscillatory shock capturing schemes II. J. Comput. Phys., 83:32-78, 1989.

J. Smagorinsky. General circulation experiments with the primitive equations, part I: The basic experiment. Mon. Weather Rev., 91:99-152, 1963.

E. Tadmor. Convergence of spectral methods for nonlinear conservation laws. SIAM J. Numer. Anal., 26:30, 1989.

G. I. Taylor. The formation of a blast wave by a very intense explosion: I, ii. 
Proc. Roy. Soc. London, Ser. A, 201:155-175, 1950.

G. I. Taylor and A. E. Green. Mechanism of the production of small eddies from large ones. Proc. Roy. Soc. A, 158:499-521, 1937.

F. A. Williams. Combustion Theory 2nd ed. Addison-Wesley, 1985. 